Gómez, J. F., Restrepo, J. E. y Díaz Larenas, C. (2021). Relación entre la disposición para comunicarse en inglés, el uso de estrategias de comunicación oral y el temor a la evaluación negativa en estudiantes universitarios. Revista de Investigación Educativa, 39(2), 411-425.

DOI: http://dx.doi.org/10.6018/rie.426741

\title{
Relación entre la disposición para comunicarse en inglés, el uso de estrategias de comunicación oral y el temor a la evaluación negativa en estudiantes universitarios
}

\section{Relationship between willingness to communicate in English, oral communication strategies use and fear of negative evaluation in university students}

\author{
Juan Fernando Gómez*, Jorge Emiro Restrepo*y Claudio Díaz Larenas** \\ *Tecnológico de Antioquia - Institución Universitaria, Colombia \\ ** Universidad de Concepción, Chile
}

\begin{abstract}
Resumen
El aprendizaje de una segunda lengua está influenciado por factores cognitivos y afectivos relacionados con el uso de estrategias, actitudes y rasgos de la personalidad que inciden en el aprendizaje. En esta investigación se analizaron las correlaciones entre la disposición para comunicarse en inglés, el uso de estrategias de comunicación para enfrentar las dificultades al hablar y el temor a la evaluación negativa en el aula, en una muestra de 575 estudiantes de pregrado de los niveles básico e intermedio de la asignatura de inglés. Se administraron el Cuestionario sobre Disposición para Comunicarse, el Inventario de Estrategias de Comunicación Oral, y la versión reducida de la Escala de Temor a la Evaluación Negativa. Se realizó una investigación cuantitativa con un diseño no experimental, correlacional y transversal. Los análisis de la prueba de Spearman revelaron que existían correlaciones positivas entre casi la totalidad de las estrategias de comunicación y la disposición para comunicarse. Se hallaron también correlaciones negativas entre la mayoría de las estrategias de comunicación y el temor a
\end{abstract}

Correspondencia: Juan Fernando Gómez, juanfer777@gmail.com. Departamento de Ciencias Básicas y Áreas Comunes. Tecnológico de Antioquia - Institución Universitaria. Calle 78B \#. 72A - 220 (Medellín - Colombia). 
la evaluación negativa. Además, se encontró una correlación débil entre el temor a la evaluación negativa y la disposición para comunicarse.

Palabras clave: competencia comunicativa; estudiante universitario; lengua inglesa; motivación; personalidad.

\section{Abstract}

Second language learning is influenced by cognitive and affective factors that are linked to the use of strategies, attitudes and personality features that affect learning. This study aimed to analyze the relationship between willingness to communicate in English, communication strategies used to cope with speaking difficulties, and fear of negative evaluation. 575 undergraduate students at the basic and intermediate levels of English took part in the study. The Willingness to Communicate Questionnaire, the Oral Communication Strategy Inventory and the Brief Fear of Negative Evaluation Scale were distributed to collect the data. The research was carried out under a correlational, cross-sectional, and non-experimental design. Spearman's correlation coefficient revealed the existence of positive correlations between the communication strategies and the willingness to communicate. Negative correlations were also found between the majority of communication strategies and fear of negative evaluation. Furthermore, a weak correlation between fear of negative evaluation and willingness to communicate was observed.

Keywords: communicative competence; undergraduate student; English language; motivation; personality.

\section{Introducción}

En la actualidad, el aprendizaje de una segunda lengua se ha convertido en una plataforma que permite el acceso a otras culturas, formas de comunicación y nuevas posibilidades laborales (Santana, García-Santillán \& Escalen-Chávez, 2016). Al respecto, Chávez-Zambrano, Saltos-Vivas \& Saltos-Dueñas (2017) argumentan que la educación superior es el ambiente en donde los estudiantes deben adquirir el dominio del idioma inglés. Aprender una nueva lengua implica el desarrollo de las habilidades básicas de escucha y expresión oral para comunicarse con otras personas, lo cual se logra a través de una pedagogía que promueve el desarrollo de la competencia comunicativa, descrita por Vorwerg (2015) como la destreza que posee un individuo para comunicarse eficazmente, lo que implica el conocimiento de los recursos comunicativos empleados por una comunidad y la habilidad para utilizarlos. Desde el origen de los enfoques basados en la competencia comunicativa en una segunda lengua, la comunicación oral se ha constituido en la meta de mayor importancia en la enseñanza de una lengua meta (Fallah, 2014).

En la institución donde se realizó la investigación se promueve el desarrollo de la competencia comunicativa en inglés bajo los modelos de enseñanza de aula invertida o 'flipped classroom' (Arnold-Garza, 2014) y el enfoque comunicativo basado en tareas 'Task Based Learning' (Zúñiga \& Gutiérrez, 2018). Por lo tanto, en las clases de inglés se realizan tareas comunicativas permanentemente, mediante presentaciones orales, y la elaboración y ejecución de un proyecto final para evidenciar el dominio oral de la lengua. En los diversos cursos de inglés se observa una alta cantidad de estudiantes que son renuentes a la realización de tareas comunicativas debido a sus limitaciones 
lingüísticas. De igual forma, una cantidad considerable manifiesta sentir temor por la evaluación que pueda emitir el docente de inglés frente al desempeño comunicativo en las actividades realizadas en clase y las evaluaciones escritas, y a los juicios negativos que podrían expresar los compañeros de clase. Por lo tanto, los aspectos mencionados anteriormente podrían afectar la disposición que un individuo pueda tener para comunicarse a través de la lengua extranjera en un contexto de aprendizaje en el cual el docente crea situaciones controladas para poner en práctica el uso del inglés.

De acuerdo con Santana et al. (2016) y Gómez (2018), existen diversas variables que inciden en el aprendizaje de una nueva lengua, como los factores cognitivos y afectivos. Los primeros comprenden la memoria, inteligencia, aptitud, y el uso de estrategias que faciliten el aprendizaje. Los segundos se refieren a las actitudes, motivaciones, creencias y rasgos de la personalidad que inciden en el aprendizaje. Este estudio se enfocó en tres de esos factores que son considerados fundamentales: disposición para comunicarse en una lengua extranjera, estrategias de comunicación para enfrentar las dificultades que surgen en la realización de tareas comunicativas en la clase de inglés y el temor a la evaluación negativa en el aula.

Teniendo en cuenta todo lo anterior, el objetivo de la presente investigación fue analizar la correlación existente entre la disposición para comunicarse en inglés, el uso de las estrategias de comunicación para enfrentar las dificultades al hablar y el temor a la evaluación negativa en el aula.

La disposición para comunicarse (DPC) es una variable que puede ayudar a comprender las razones por las cuales un individuo no desea hablar en inglés en el aula (Arpaci-Somuncu, 2016). Es un elemento de alta importancia porque mejora el aprendizaje de la segunda lengua (Yaraghi \& Shafiee, 2018; Makiabadi, Pishghadam, Meidani \& G.H., 2019) ya que es un aspecto que influye en la producción oral (Philp \& Gurzynski-Weiss, 2020). La DPC es un constructo complejo en el que están involucrados factores lingüísticos, psicológicos y culturales (Asmalı, 2016). Esta variable motivacional hace referencia a la intención que tiene un individuo para participar en una conversación en la lengua extranjera de manera verbal, cuando tiene la posibilidad de hacerlo libremente. Según Wood (2016), la disposición para comunicarse se configura como un estado mental que ocasiona que un individuo se incline a tomar parte en una conversación con un interlocutor específico, en un momento determinado, dado el estado de autoconfianza para asumir el rol de emisor.

Arpaci-Somuncu (2016) y Philp \& Gurzynski-Weiss (2020) explican que la DPC depende de los rasgos de la personalidad (introversión, ansiedad o temor para comunicarse, auto percepción sobre la competencia en la lengua meta y la autoestima), y de aspectos situacionales (número de personas, grado de familiaridad, grado de la evaluación emitida y el tema). En consideración a lo anterior, podría decirse que la disposición para comunicarse es un factor individual en donde convergen experiencias, particularidades de los contextos comunicativos, la competencia en la lengua meta percibida por el individuo y una variedad de características socio-psicológicas que influyen el desempeño verbal durante la interacción.

En cuanto a las Estrategias de Comunicación Oral (ECO), diversos autores argumentan que estas pueden ayudar a los estudiantes a compensar las deficiencias en la lengua meta y desarrollar con mayor efectividad tareas que implican la comunicación 
oral en el aula, pese a las limitaciones lingüísticas (Jindathai, 2017; Zhou \& Huang, 2018). Estas estrategias son concebidas por Nakatani (2010) como intentos que realizan los estudiantes para resolver los problemas en la interacción, mejorar la comunicación con el interlocutor y al mismo tiempo, lograr un mejor desarrollo de la habilidad oral del inglés como lengua extranjera. Los comportamientos asumidos por los estudiantes durante la interacción se constituyen en estrategias verbales y no-verbales que les permiten lograr el propósito comunicativo.

En la realización de tareas que implican la interacción en el aula, hay estudiantes que suspenden su intento para comunicarse debido a limitaciones o al desconocimiento de los recursos lingüísticos de la lengua meta, los cuales comprenden los siguientes elementos: gramática, léxico, morfología, sintaxis, entonación y pronunciación. Por lo tanto, las ECO operan como un recurso que permite mantener la fluidez en la comunicación y compensar sus vacíos.

En lo concerniente al temor a la evaluación negativa (TEN), esta es definida por Yokus (2013) como un estado de ansiedad y temor que experimenta una persona en las relaciones interpersonales o situaciones sociales ya que cree que será juzgado negativamente. En el aula, los estudiantes pueden experimentar ambas sensaciones, puesto que allí se evalúan, de diferentes formas, las prácticas de uso de la lengua, que son enseñadas y controladas por los docentes. La ansiedad es un elemento que obstruye el aprendizaje de una lengua extranjera. Al respecto, Ortega (2014) afirma que la ansiedad originada al momento de hablar en inglés evidencia diversos síntomas, pero lo más comunes son el bloqueo cuando se solicita expresarse en frente de los demás en clase y el confundirse al momento de responder una prueba escrita a pesar de haberla preparado, e incluso saber las respuestas. Por su parte, Idri (2011) indica que las mayores fuentes de temor a la evaluación negativa provienen de los docentes y los pares en el momento de la interacción y de la corrección de los errores. En el aula, los estudiantes son constantemente monitoreados y evaluados por el docente de lengua extranjera y de forma indirecta por los otros pares, lo que origina un mayor estado de ansiedad y temor cuando se presenta el momento de evaluar el desempeño comunicativo.

Se realizó una revisión exhaustiva de la literatura en algunas en las principales bases de datos, pero no se encontró ningún estudio que conjugara las tres variables que se abordaron en esta investigación. Algunos de los resultados más significativos en trabajos que incluyeron las variables en mención, en contextos de aprendizaje del inglés como lengua extranjera, se presentan a continuación. Yaraghi \& Shafiee (2018) investigaron las posibles relaciones entre la autonomía del estudiante (AE), la disposición para comunicarse (DPC) y las estrategias de comunicación oral (ECO), además de un análisis de la DPC y la AE como predictores del uso de las ECO, con una población de 102 estudiantes de inglés de una universidad privada de Irán. Se encontró una correlación débil positiva entre la DPC y el uso de las ECO. Además, se halló que la DPC es el mejor predictor de las ECO, con relación a la AE, puesto que predijo el uso de 6 factores de los 8 que componen las estrategias de comunicación oral. Por otro lado, la AE fue un predictor significativo del factor de las estrategias socioafectivas. Se concluyó que hay diversos aspectos que influyen en la DPC, como las situaciones de la clase, los factores individuales, el contexto y aspectos lingüísticos. 
En el contexto de indonesia, Manipuspika (2018) realizó un estudio con 98 estudiantes de inglés en una universidad pública, en el cual propuso identificar el tipo y nivel de ansiedad experimentada por los participantes y comprobar las correlaciones entre el nivel de la ansiedad en el aprendizaje de una lengua extranjera (ALE) y la disposición para comunicarse (DPC). Se reportó una correlación positiva fuerte entre la ALE y DPC. Los tipos de ansiedad encontrados correspondieron al temor a la evaluación negativa, la ansiedad frente a los exámenes y la aprehensión comunicativa (temor ante situaciones de comunicación). Los estudiantes tuvieron un nivel de ansiedad alto y por esta razón se les hizo difícil el proceso de aprendizaje y demostraron un bajo deseo de hablar en la lengua meta.

Por su parte, Šafranj \& Zivlak (2019) examinaron la manera en que la ansiedad en el aprendizaje de una lengua extranjera (ALE) está condicionada por el temor a la evaluación negativa (TEN) y por ciertos rasgos de la personalidad. Se aplicó el instrumento 'Five Factor Model' (FFM) adaptado por Goldberg (1992), con una muestra de 296 estudiantes universitarios en Serbia. Los hallazgos de mayor relevancia evidenciaron que los bajos niveles de estabilidad emocional fueron un predictor consistente de los altos niveles de la ALE y sus tipos dentro de las escalas y subescalas que la conforman. Asimismo, los estudiantes propensos a sentir temor a la evaluación negativa estuvieron más inclinados a experimentar ansiedad. La correlación entre TEN y el temor a la retroalimentación por pares y docentes resultó ser positiva moderada.

\section{Método}

Se realizó una investigación cuantitativa con diseño no experimental, de nivel correlacional y de corte transversal a través de la cual se determinaron los grados de correlación entre las variables 'Disposición para comunicarse' (DPC), 'Estrategias de Comunicación Oral' (ECO) y Temor a la Evaluación Negativa (TEN).

\section{Objetivo}

Este trabajo planteó como objetivo analizar la correlación existente entre la disposición para comunicarse en inglés, el uso de las estrategias de comunicación para enfrentar las dificultades al hablar y el temor a la evaluación negativa en el aula.

\section{Población y muestra}

La muestra del estudio estuvo conformada por 575 estudiantes de pregrado de los programas de psicología, negocios internacionales, trabajo social, licenciatura en educación preescolar y gestión comercial, quienes estaban matriculados en el Tecnológico de Antioquia - Institución Universitaria, una universidad pública. Los participantes fueron seleccionados de manera aleatoria entre 46 cursos. Se incluyeron 163 hombres $(28,3 \%)$ y 412 mujeres $(71,7 \%)$, distribuidos en tres estratos poblacionales de acuerdo al nivel de dominio de la lengua extranjera, según el número de horas aprobadas en los cursos y los lineamientos del Consejo de Europa (2002): el primero (A1 - curso inglés 1 y 2) conformado por 202 estudiantes, el segundo (A2 - curso inglés 3 y 4) constituido 
por 201 y el tercero (B1 - curso inglés 5 y 6) comprendió 172 individuos. El rango de edad en los encuestados se encuentra entre los 15 y 54 años, con una media de 23,1.

\section{Instrumentos}

Los datos fueron obtenidos a través de tres instrumentos, que se aplicaron de forma presencial, en una sola sesión en una sala de sistemas donde, utilizando computadores, los estudiantes respondieron cada instrumento. La recolección de datos fue realizada durante los primeros meses del año 2020. El primer instrumento correspondió al Cuestionario sobre Disposición para Comunicarse, traducido por Díaz (2006), el cual contiene diez afirmaciones que corresponden a situaciones en las que un estudiante estaría dispuesto a comunicarse en la lengua meta en el aula. Esta versión descarta algunas afirmaciones por ser consideradas por el autor como reiterativas. La versión original de este instrumento fue creada por McCroskey \& Richmond (1990) y comprende veinte situaciones. Cada aseveración fue ponderada a partir de la escala Likert de la siguiente manera: Nunca (1), Casi nunca (2), Alguna vez (3), Mitad del tiempo (4), Normalmente (5) y Casi siempre (6). Los rangos de puntuación van de 0 a 50 puntos. La versión original está compuesta por siete factores cuyos ítems tienen una consistencia interna que fluctúa entre 0.65 y 0.92 (alfa de Cronbach). De acuerdo con los autores, la confiabilidad total del instrumento es de 0.92 y tiene una fuerte validez de contenido. También informan que hay evidencia de validez de constructo y validez predictiva (McCroskey \& Richmond, 1990).

La segunda fuente de datos correspondió a la aplicación del Inventario de Estrategias de Comunicación Oral, creado por Nakatani (2006), empleado para medir la frecuencia del uso de las estrategias para enfrentar las dificultades en la comunicación oral y en la comprensión auditiva. Se aclara que sólo se abordó la habilidad de la expresión oral. El cuestionario comprende los siguientes factores: estrategias socioafectivas, estrategias orientadas hacia la fluidez, estrategias negociación del significado, estrategias orientadas hacia la exactitud, estrategias de reducción y alteración del mensaje, estrategias no-verbales, estrategias de abandono del mensaje y estrategias de intento de pensar en inglés. Se emplearon los rangos propuestos por Yaman \& Özcan (2015), para determinar la frecuencia de uso las estrategias en la comunicación oral, según los siguientes valores: 1.0 - 2.49 (Baja), 2.5 - 3.49 (Media) y 3.5 - 5.0 (Alta). De acuerdo con Nakatani (2006), la consistencia interna total fue de 0.86 (alfa de Cronbach), se presentó una adecuada estructura factorial de ocho factores y se comprobó su validez concurrente.

El tercer instrumento de recolección de datos correspondió a Escala de Temor a la Evaluación Negativa. Esta herramienta fue desarrollada por Leary (1983) y evalúa la intensidad del temor que una persona puede experimentar ante la posibilidad de ser juzgada negativamente por otros individuos. Consiste en doce ítems que son respondidos a partir de un formato de escala tipo Likert de la siguiente manera: (1) Nada característico en mí, (2) Ligeramente característico en mí, (3) Moderadamente característico en mí, (4) Muy característico en mí, (5) extremadamente característico en mí. Los ítems 2, 4, 7 y 10 son calificados de manera inversa. En el presente estudio se empleó la versión de la escala propuesta por Gallego, Botella, Quero, Baños \& García-palacios (2007). Según estos autores, el instrumento tiene una adecuada estructura bifactorial, una consistencia interna total de .92 (alfa de Cronbach) y adecuados valores de validez convergente y discriminante. 


\section{Procedimiento de recogida y análisis de datos}

Para la administración de los diferentes instrumentos se contó con la aprobación de los equipos directivos del centro educativo. Los datos fueron analizados a partir del coeficiente de correlación de Spearman, puesto que la prueba de normalidad SmirnovKolmogorov determinó que no siguen una distribución normal.

\section{Resultados}

La prueba de Spearman fue aplicada de manera separada entre cada uno de los factores que componen la variable 'Estrategias de Comunicación Oral' (ECO) y la variable unidimensional 'Disposición para Comunicarse' (DPC) para identificar la intensidad de asociación existente entre ellas. Se siguió la propuesta de Mukaka (2012) para interpretar el grado de correlación. De acuerdo con la Tabla 1 , se evidenció una correlación positiva moderada entre las estrategias socioafectivas y la DPC $(\mathrm{r}=.511, \mathrm{p}<.000)$, entre las orientadas hacia la fluidez y la DPC $(\mathrm{r}$ $=.448, \mathrm{p}<.000)$, entre el de negociación del significado y la DPC $(\mathrm{r}=.438, \mathrm{p}<.000)$, $\mathrm{y}$ entre las orientadas hacia la exactitud y la DPC $(\mathrm{r}=.406, \mathrm{p}<.000)$. Además, se halló una correlación positiva débil entre las estrategias de reducción y alteración del mensaje y la DPC $(\mathrm{r}=.133, \mathrm{p}<.001)$, entre las estrategias no verbales y la DPC $(\mathrm{r}=.286, \mathrm{p}<.000)$. Adicionalmente, se identificó una relación negativa débil entre las de abandono del mensaje y la DPC $(\mathrm{r}=-.280$, $\mathrm{p}<.000)$. No se encontró ninguna correlación concerniente al factor de las estrategias de intento de pensar en inglés y la DPC $(\mathrm{r}=-.020, \mathrm{p}<.627)($ Ver Tabla 1$)$.

Tabla 1

Correlaciones entre las variables Estrategias de Comunicación Oral (ECO) y Disposición para Comunicarse $(D P C)$

\begin{tabular}{lll}
\hline ECO/DPC & Coeficiente & P \\
\hline E. Socioafectivas/DPC & .511 & $.000^{* *}$ \\
E. Orientadas hacia la fluidez/DPC & .448 & $.000^{* *}$ \\
E. Negociación del significado/DPC & .438 & $.000^{* *}$ \\
E. Orientadas hacia la exactitud/DPC & .406 & $.000^{* *}$ \\
E. Reducción y alteración del mensaje/DPC & .133 & $.001^{* *}$ \\
E. No verbales/DPC & .286 & $.000^{* *}$ \\
E. Abandono del mensaje/DPC & -.280 & $.000^{* *}$ \\
E. Intento de pensar en inglés/DPC & -.020 & .627 \\
\hline
\end{tabular}

** Correlación significativa para $\mathrm{p}<.01$

Fuente: elaboración de los autores 
Como se observa en la Tabla 2 más abajo, la prueba de Spearman fue utilizada de forma independiente entre las variables que conforman el instrumento ECO y el Temor a la Evaluación Negativa (TEN), para determinar el grado de asociación entre ambas. Se encontró una correlación negativa débil entre las estrategias socioafectivas y el TEN ( $\mathrm{r}=-.286, \mathrm{p}<.000)$, entre las orientadas hacia la fluidez y el TEN $(\mathrm{r}=-.189$, $\mathrm{p}<.000)$, entre las de negociación de significado $(\mathrm{r}=-.171, \mathrm{p}<.000)$, entre las orientadas hacia la exactitud $(-.115, \mathrm{p}<.006)$, y de igual modo, entre las estrategias no verbales y el TEN ( $\mathrm{r}=-.138, \mathrm{p}<.001)$. Adicionalmente, el test estadístico aplicado demostró la existencia de una correlación positiva débil entre las de abandono del mensaje y el TEN ( $\mathrm{r}=.361, \mathrm{p}<.000)$. En lo concerniente a las estrategias de reducción y alteración del mensaje y su relación con el TEN no se reportó ninguna correlación, puesto que el resultado fue $\mathrm{r}=.028, \mathrm{p}<.509$. Del mismo modo, no se presentó ninguna correlación entre las de intento de pensar en inglés y el TEN, dado que $\mathrm{r}=.082, \mathrm{p}<.050$.

Tabla 2

Correlaciones entre las variables Estrategias (ECO) y Temor a la evaluación negativa (TEN).

\begin{tabular}{lcc}
\hline ECO/TEN & Coeficiente & $\mathbf{P}$ \\
\hline E. Socioafectivas/TEN & -.286 & $.000^{* *}$ \\
E. Orientadas hacia la fluidez/TEN & -.189 & $.000^{* *}$ \\
E. Negociación del significado/TEN & -.171 & $.000^{* *}$ \\
E. Orientadas hacia la exactitud/TEN & -.115 & $.006^{* *}$ \\
E. Reducción y alteración del mensaje/TEN & .028 & $.509^{* *}$ \\
E. No verbales/TEN & -.138 & $.001^{* *}$ \\
E. Abandono del mensaje/TEN & .361 & $.000^{* *}$ \\
E. Intento de pensar en inglés/TEN & .082 & .050 \\
\hline
\end{tabular}

* Correlación significativa para $\mathrm{p}<.05$

** Correlación significativa para $\mathrm{p}<.01$

Fuente: elaboración de los autores

La Tabla 3 muestra que existe una relación débil negativa entre la DPC y el TEN ( $\mathrm{r}$ $=-.389, \mathrm{p}<.000)$. A pesar de que es una correlación débil, este valor demuestra que, a mayor temor a la evaluación negativa, habrá una menor disposición para realizar las tareas comunicativas en el aula.

Tabla 3

Correlaciones entre las variables Disposición para comunicarse en inglés (DPC) y Temor a la evaluación negativa (TEN).

\begin{tabular}{lll}
\hline DPC/TEN & Coeficiente & P \\
\hline E. Socioafectivas/TEN & -.340 & $.000^{* *}$ \\
\hline ** Correlación significativa para $p<.01$ & & \\
Fuente: elaboración de los autores. & &
\end{tabular}




\section{Discusión y conclusiones}

En esta investigación se propuso analizar la correlación existente entre la disposición para comunicarse en inglés, el uso de las estrategias de comunicación para enfrentar las dificultades al hablar y el temor a la evaluación negativa en el aula. En primer lugar, La prueba de Spearman demostró una correlación positiva moderada entre el uso de la mayoría de las estrategias de comunicación oral y la DPC en inglés. Esto indica que la disposición de los estudiantes para hablar es incrementada a medida que emplean las ECO, lo que resulta consistente con los hallazgos de Yousef, Jamil, \& Razak (2013), y está en oposición con lo reportado por Yaraghi \& Shafiee (2018). Además, estas estrategias se constituyen en un elemento que ayuda a los estudiantes a resolver problemas en la comunicación causados por las insuficiencias lingüísticas (Jindathai, 2017). De acuerdo con Cameron (2015), la motivación y otros factores personales ejercen un impacto positivo que incrementa la disposición para comunicarse, y las ECO estimulan la motivación, la auto percepción sobre la competencia en la lengua meta y la DPC.

La correlación más fuerte se presentó entre las estrategias socioafectivas y la DPC. Según Yaraghi \& Shafiee (2018), esto indica que aquellos que están dispuestos a comunicarse en una segunda lengua recurren a estas estrategias para dar una buena impresión al interlocutor y evitar el silencio en el momento de la interacción. Además, esta es una manera de controlar las emociones, reducir la ansiedad, e inclusive auto animarse para lograr el objetivo comunicativo, sin importar los errores que puedan cometerse. En este sentido, Ata Allah (2016) sustenta que estas estrategias pueden facilitar el aprendizaje de una segunda lengua y que pueden contribuir a incrementar la competencia lingüística.

La correlación entre las estrategias orientadas hacia la fluidez y la DPC también fue positiva moderada. Por lo tanto, aquellos estudiantes que están dispuestos a comunicarse prestan atención a la pronunciación, a la claridad del discurso y se toman su tiempo para evitar expresar mensajes erróneos a los interlocutores. De acuerdo con Mariani (2010), existe una dimensión de la personalidad que influye en la elección de una estrategia sobre otra y que ocasiona que algunas personas presten mayor importancia a la comunicación, y otros se encausen hacia la forma del lenguaje. La revisión de la literatura realizada por Rahmawati \& Nurmayasari (2014) confirma que el tipo de personalidad (extrovertidos e introvertidos) afecta la manera en que aprenden los estudiantes y que de igual modo existe una relación entre ambas y la habilidad del habla en una lengua extranjera.

Por otro lado, la correlación entre las estrategias de negociación del significado y la DPC resultó ser positiva moderada. Esto significa que estos estudiantes enfrentan las dificultades que surgen en la comunicación oral por medio de la negociación, y la emplean para reducir la ansiedad (Metcalfe \& Noom-Ura, 2013), permiten mantener la comunicación (Arpaci-Somuncu, 2016) y solucionar los problemas que pueden generase debido al desconocimiento de vocabulario. Este resultado es afín con Yaraghi \& Shafiee (2018), aunque discordante con Yousef et al. (2013), puesto que estas estrategias fueron las que mayor impacto tuvieron sobre la DPC. Najafabadi (2014) sostiene que estas estrategias son utilizadas al momento de confirmar 
la comprensión del interlocutor y permiten la inclusión de ejemplos para confirmar que el mensaje ha sido lo suficientemente claro.

La correlación entre las estrategias orientadas hacia la exactitud y la DPC correspondió a positiva moderada, las de abandono del mensaje y la DPC indicaron ser negativa débil, mientras que las no verbales y la DPC se ubicaron en el rango de positiva débil. Por otro lado, se descubrió una correlación positiva débil entre las estrategias de reducción y alteración del mensaje y la DPC. Estos resultados son afines con lo reportado en la investigación de Yaraghi \& Shafiee (2018). Esto significa que aquellos que poseen una mayor DPC en la lengua extranjera recurren a emplear las formas gramaticales adecuadas, se autocorrigen, usan expresiones faciales y gestos para darle pistas al interlocutor para que comprenda el mensaje. Del mismo modo, cuando los más dispuestos tienen dificultades para ejecutar el plan verbal que han concebido en su mente debido a la insuficiencia lingüística, recurren a solicitar ayuda de sus compañeros para evitar abandonar su intento comunicativo.

El segundo hallazgo demostró que en esta muestra las estrategias ECO ejercen un impacto leve en la reducción del temor a la evaluación negativa, dado que se evidenciaron correlaciones negativas débiles entre la mayoría de las estrategias de comunicación oral y el TEN. Este resultado se encuentra alineado con Liu \& Tarnopolsky (2018), aunque es opuesto a los hallazgos de Tian \& Mahmud (2018), dado que las correlaciones reportadas fueron en su mayoría positivas. Debido al grado de correlación, las estrategias más notables utilizadas para reducir el TEN resultaron ser en, primer lugar, las de abandono del mensaje y, en segundo, las socioafectivas. Esto significa que los estudiantes enfrentan el temor a la evaluación negativa ocasionada por las insuficiencias lingüísticas en la lengua meta a partir de la simplificación del mensaje que se desea expresar, a través de la dicción de frases simples y palabras que les son familiares, para lograr transmitir el plan verbal concebido en la mente. Además, tratan de relajarse para reducir la ansiedad y las actitudes negativas, para favorecer el propósito comunicativo.

De acuerdo con Atma \& Nosmalasari (2016), las dificultades para hablar con los demás en una lengua extranjera son un indicador de la ansiedad y de la aprehensión para comunicarse de manera oral. De acuerdo con Harris (2016), esta última se define como una respuesta psicológica al ser evaluado, la cual se convierte rápidamente en una repercusión física, puesto que el cuerpo responde a la amenaza que la mente percibe. Esto permite pensar que los estudiantes de esta muestra experimentan temor a la evaluación negativa por estar más preocupados por la manera en que se corregirán los errores que puedan cometer durante la producción del discurso, que por aquellos errores que deberían ser necesariamente corregidos para mejorar la producción oral.

Como tercer hallazgo, se descubrió una correlación negativa débil entre la DPC y el TEN. Este resultado se encuentra alineado con la idea que plantea Liu \& Tarnopolsky (2018), en la cual expone que la ansiedad en la lengua extranjera está asociada con el temor a la evaluación negativa y el temor a cometer errores. Por lo tanto, un estudiante que se encuentra bajo este estado se desanima y su nivel de disposición para comunicarse disminuye. Este resultado refleja que, en cierto grado, el temor a la evaluación negativa influye en la disposición para comunicarse en la legua extranjera, lo cual es consistente con Rastegar \& Karami (2015) y Manipuspika (2018). En cuanto 
a la comunicación en una lengua extranjera, es notable que en el contexto del estudio un alto número de personas evitan la comunicación oral. Según Rastegar \& Karami (2015), esto podría ocurrir debido a que se genera un tipo de ansiedad durante el aprendizaje de una lengua y un individuo que teme ser evaluado negativamente tiene mayor tendencia a estar más nervioso para hablar, máxime cuando siente angustia debido a su inhabilidad lingüística.

En esta investigación se propuso analizar la correlación existente entre la disposición para comunicarse en inglés, el uso de las estrategias de comunicación para enfrentar las dificultades al hablar y el temor a la evaluación negativa en el aula. En primera instancia, se encontró que existe una asociación significativa entre el uso de la mayoría de las estrategias de comunicación oral y la DPC. En segundo lugar, se observaron correlaciones negativas entre la mayoría de las estrategias de comunicación y el temor a la evaluación negativa. Por último, se descubrió una correlación débil entre el temor a la evaluación negativa y la disposición para comunicarse. Lo anterior significa que la DPC impacta en el uso de las estrategias ECO, el temor a la evaluación negativa es de cierto modo reducido por las ECO, y en cierto grado, el temor a la evaluación negativa ejerce una influencia en la disposición para comunicarse en la legua extranjera.

La DPC es un rasgo psicológico determinado por múltiples factores (MacIntyre, 1994). La personalidad es, quizás, uno de los más importantes y la autoconfianza es otro igualmente relevante. Evidentemente, los factores situacionales influyen en la disposición para comunicarse, pero estos dos aspectos psicológicos son clave para entender por qué unas personas, más que otras, tienen una tendencia hacia la interacción comunicativa verbal. En el contexto del aprendizaje del inglés, la competencia lingüística es tan importante como la motivación para el aprendizaje, la autoconfianza y el interés por la interacción verbal. Estos tres últimos corresponden a la dimensión actitudinal y no a la aptitudinal, como lo es el primero.

Cuando las aptitudes lingüísticas son insuficientes (deficiencias en la gramática, el léxico, la morfología, la sintaxis, la entonación o la pronunciación) o están en proceso de adquisición, los estudiantes recurren a ciertas estrategias comunicativas. Sin embargo, su uso está asociado, como quedó demostrado con los resultados del presente estudio, con factores psicológicos, como la disposición para comunicarse (que tiene una estrecha vinculación con el estilo de personalidad) y con el temor a la evaluación negativa (que está asociado con factores cognitivos, como creencias sobre la forma como los demás evaluarán el desempeño; y auto esquemas, como la autoeficacia, que es una medida de qué tanta seguridad se tiene sobre las propias capacidades).

De esta investigación se derivan ciertas implicaciones pedagógicas. Primero, se considera que los docentes de inglés pueden ejercer un impacto positivo en los estudiantes con relación a la DPC. Cuando un docente valora la participación de los estudiantes, los anima con palabras positivas, promueve un ambiente de respeto, genera seguridad y confianza en ellos mismos, y los alienta a participar en un espacio rico en experiencias comunicativas similares a las que viven en la vida real, es más probable que la motivación de los estudiantes aumente, al igual que su DPC. Los estudiantes con mayores niveles de temor a la evaluación negativa tenderán a tener menor disposición para comunicarse. Ciertamente, no querrán exponerse a la crítica y la burla de sus compañeros. Estos estudiantes tienen mayor aprehensión emocional y requerirán 
que el docente utilice estrategias pedagógicas que propicien un ambiente sin tensiones que permita reducir su ansiedad. Como lo mostraron los resultados, la aprehensión emocional (temor a la evaluación negativa) correlaciona negativamente con casi todas las estrategias de comunicación oral. Esto es, a mayor temor, menor uso de las estrategias.

En segundo término, Atma \& Nosmalasari (2016) sustentan que las dificultades en la comunicación oral están ligadas al empleo de las ECO, dado que estas son precisamente utilizadas para resolverlas. Por esta razón, es el docente quien puede ayudar a los estudiantes a tomar conciencia de como emplearlas para evitar dejar los mensajes inconclusos o renunciar al intento comunicativo, y poder mejorar el desarrollo en la producción durante la realización de tareas comunicativas en el aula. Tian \& Mahmud (2018) explican que los estudiantes deben tener una instrucción directa sobre el uso de las estrategias de comunicación para evitar el impacto negativo que produce la ansiedad al hablar en público en la lengua meta

Este estudio presentó algunas limitaciones que es necesario enunciar. Primero, las apreciaciones de los estudiantes frente a las variables abordadas en este estudio no fueron consideradas, y la única fuente de información correspondió a los tres cuestionarios aplicados. La comprensión del fenómeno estudiado podría ampliarse si se triangulan los resultados con grabaciones que registren el comportamiento de los estudiantes durante la realización de tareas comunicativas en el aula, y con entrevistas individuales. Segundo, este estudio solo se enfocó en el análisis y discusión de resultados que pueden ser generalizados exclusivamente para el contexto del Tecnológico de Antioquia. Por lo tanto, concebir una investigación a nivel regional con poblaciones universitarias en instituciones públicas y privadas, podría generar resultados que proporcionen nueva información.

Finalmente, se proponen las siguientes líneas de investigación futuras en concordancia con el tema abordado en este estudio: 1. Incluir variables como el sexo, nivel de dominio y grado de ansiedad de los estudiantes. De este modo, se podrían explorar relaciones entre estas variables y el uso de las estrategias de comunicación oral y de comprensión auditiva. 2. Analizar los efectos que podría producir la corrección de los errores en cuanto a la manera (implícita o explícita), y respecto al tiempo (inmediata o tardía), con relación al impacto que causa en la DPC y al empleo de las ECO.

\section{Referencias}

Arnold-Garza, S. (2014). The flipped classroom teaching model and its use for information literacy instruction. Communications in Information Literacy, 8(1), 7-22. http:// dx.doi.org/10.15760/comminfolit.2014.8.1.161

Arpaci-Somuncu, D. (2016). Turkish EFL learners' use of communication strategies and its predictors. ELT Research Journal, 5(3), 178-192.

Asmalı, M. (2016). Willingness to communicate of foreign language learners in Turkish context. Procedia - Social and Behavioral Sciences, 232, 188-195. http://doi: 10.1016/j. sbspro.2016.10.044

Ata Allah, T. (2016). Social-affective strategy instruction in EFL materials: the case of English textbooks and official programs in Tunisia. International Journal of Humanities and Cultural Studies, 2(4), 169-195. 
Atma, N. y Nosmalasari, N. (2016). Communication strategies: do they differ across the students' level of language learning anxiety? Proceedings of ISELT FBS. Universitas Negeri Padang, 4(2), 162-169.

Cameron, D. (2015). 'In New Zealand I feel more confidence': the role of context in the willingness to communicate (WTC) of migrant Iranian English language learners. International Journal of English Studies, 15(2), 61-80. http://doi: 10.6018/ijes/2015/2/202981

Consejo de Europa (2002). Marco común europeo de referencia para las lenguas: aprendizaje, enseñanza, evaluación. (Traducido por el Instituto Cervantes 2002) Madrid: Anaya.

Chávez-Zambrano, M. X., Saltos-Vivas, M. A. y Saltos-Dueñas, C. M. (2017). La importancia del aprendizaje y el conocimiento del idioma inglés en la enseñanza superior. Dominio de Las Ciencias, 3, 759-771. http://doi:10.23857/dc.v3i3\%20mon.707

Díaz, E. R. (2006). Estudio sobre las inteligencias inter- e intrapersonales como instrumentos de desarrollo de la disposición a comunicarse en el aula. Universidad de Huelva. Recuperado de http://rabida.uhu.es/dspace/handle/10272/157

Fallah, N. (2014). Willingness to communicate in English, communication self-confidence, motivation, shyness and teacher immediacy among Iranian English-major undergraduates: a structural equation modeling approach. Learning and Individual Differences, 30, 140-147. http://doi: 10.1016/j.lindif.2013.12.006

Gallego, M. J., Botella, C., Quero, S., Baños, R. M. y García-palacios, A. (2007). Propiedades psicométricas de la escala de miedo a la evaluación negativa versión breve (BFNE) en muestra clínica. Revista de Psicopatología y Psicología Clínica, 12(3), 163-176.

Goldberg, L. R. (1992). The Development of markers for the Big-Five factor structure. Psychological Assessment, 4(1), 26-42. https://doi:10.1037/1040-3590.4.1.26

Gómez, J. F. (2018). Diferencias en las creencias entre hombres y mujeres acerca del aprendizaje del idioma inglés. Revista Signos Estudios de Lingüística, 51(97), 193-213. http://doi: 10.4067/S0718-09342018000200193

Harris, L. J. (2016). Stand up, speak out: the practice and ethics of public speaking. Milwaukee: Communication Faculty Book.

Idri, N. (2011). Foreign language anxiety among Algerian EFL students: the case of first year students of English at the university of Abderahmane Mira-Béjaia; LMD (Licence/Master/Doctorat) system group. Universal Journal of Education and General Studies, 1(3), 55-64.

Jindathai, S. (2017). Communication strategies of Engineering students at a private university institute in Bangkok in the academic year of 2015. The New English Teacher, 11(1), 197-216.

Leary, M. R. (1983). A brief version of the fear of negative evaluation scale. Personality and Social Psychology Bulletin, 9(3), 371-375.

Liu, M. y Tarnopolsky, O. (2018). Interactive effects of English-speaking anxiety and strategy use on oral English test performance of high- and low-proficient Chinese university EFL learners. Cogent Education, 5(1), 1-14. http://doi: 10.1080/2331186X.2018.1562410

MacIntyre, P. D. (1994). Variables underlying willingness to communicate: A causal analysis. Communication Research Reports, 11(2), 135-142, http://doi: $10.1080 / 08824099409359951$ 
Makiabadi, H., Pishghadam, R., Meidani, E. N. y G.H., K. (2019). Examinar el papel de la «emotioncy» en la voluntad de comunicarse: un enfoque de modelos de ecuaciones estructurales. Revista de Psicodidáctica, 24(2), 120-130. http://doi: 10.1016/j. psicod.2019.03.002

Manipuspika, Y. S. (2018). Correlation between anxiety and willingness to communicate in the Indonesian EFL context. Arab World English Journal, 9(2), 200-217. http://doi: 10.24093/awej/vo19no2.14

Mariani, L. (2010). Communication strategies: learning and teaching how to manage oral interaction. Italy: Learning Paths-Tante Vie Per Imparare. http://doi: 10.1093/elt/ccs026

McCroskey, J. C. y Richmond, V. P. (1990). Willingness to communicate: A cognitive view. Journal of Social Behavior and Personality, 5(2), 19-37.

Metcalfe, J. y Noom-Ura, S. (2013). Communication strategy use of high and low proficiency learners of English at a Thai university. Learn Journal, 6(1), 66-87.

Mukaka, M. M. (2012). Statistics corner: a guide to appropriate use of correlation coefficient in medical research. Malawi Medical Journal, 24(3), 69-71.

Najafabadi, N. K. (2014). The use of speaking strategies by Iranian EFL university students. International Journal of Foreign Language Teaching and Research, 2(5), 11-25.

Nakatani, Y. (2006). Developing an oral communication strategy inventory. Modern Language Journal, 90(2), 151-168. http://doi: 10.1111/j.1540-4781.2006.00390.x

Nakatani, Y. (2010). Identifying strategies that facilitate EFL learners' oral communication: a class room study using multiple data collection procedures. Modern Language Journal, 94(1), 116-136. http://doi: 10.1111/j.1540-4781.2006.00390.x

Ortega, L. (2014). Understanding second language acquisition. (B. Comrie y G. Corbett, Eds.). New York: Routledge. http://doi: 10.1017/CBO9781107415324.004

Philp, J. y Gurzynski-Weiss, L. (2020). On the role of the interlocutor in second language development: a cognitive-interactionist approach. In L. Gurzynski-Weiss (Ed.), Crosstheoretical explorations of interlocutors and their individual differences (pp. 19-50). Amsterdam, The Netherlands: John Benjamin's Publishing Company.

Rahmawati, S. y Nurmayasari, E. (2014). A comparative study between extroverted and introverted students and their speaking ability. English Journal, 15(2), 20-29.

Rastegar, M. y Karami, M. (2015). On the relationship between foreign language classroom anxiety, willingness to communicate and scholastic success among Iranian EFL learners. Theory and Practice in Language Studies, 5(11), 2387-2394. http://doi: 10.17507/tpls.0511.25

Šafranj, J. y Zivlak, J. (2019). Effects of big five personality traits and fear of negative evaluation on foreign language anxiety. Croatian Journal of Education, 21(1), 275-306. http://doi: 10.15516/cje.v21i1.2942

Santana, J., García-Santillán, A. y Escalen-Chávez, M. (2016). Variables que influyen sobre el aprendizaje del inglés como segunda lengua. Revista Internacional de Lenguas Extranjeras, 5, 79-94. http://doi: 10.17345/rile201679-94

Tian, S. y Mahmud, M. (2018). A study of academic oral presentation anxiety and strategy employment of EFL graduate students. Indonesian Journal of EFL and Linguistics, 3(2), 149-170. http://doi: 10.21462/ijefl.v3i2.78 
Vorwerg, C. (2015). Communicative competence: linguistic aspects. In International Encyclopedia of the Social and Behavioral Sciences (2nd ed., Vol. 4, pp. 294-301). http:// doi: 10.1016/B978-0-08-097086-8.53042-6

Wood, D. (2016). Willingness to communicate and second language speech fluency: an idiodynamic investigation. System, 60, 11-28. http://doi: 10.1016/j.system.2016.05.003

Yaman, Ş. y Özcan, M. (2015). Oral communication strategies used by Turkish students learning English as a foreign language. In M. Pawlak and E. Waniek-Klimczak (eds.) (Ed.), Issues in Teaching, Learning and Testing Speaking in a Second Language (pp. 143-158). New York: Springer-Verlag.

Yaraghi, E. y Shafiee, S. (2018). Roles of learner autonomy and willingness to communicate in communication strategy use of EFL learners. International Journal of English Language and Literature, 7(3), 55-74. http://doi: 10.18488/journal.23.2018.73.55.74

Yokus, H. (2013). Investigation of relationship between the fear of negative evaluation and musical instrument achievements of music teacher candidates. International Journal of New Trends in Arts, Sports y Science Education, 2(3), 16-22.

Yousef, R., Jamil, H. y Razak, N. (2013). Willingness to communicate in English: a study of Malaysian pre-Service English teachers. English Language Teaching, 6(9), 205-216. http://doi: 10.5539/elt.v6n9p205

Zhou, J. y Huang, L.-S. (2018). An exploration of strategies used by Chinese graduate students in electrical engineering and education: integrating questionnaire, task performance, and post-task recall data. Asian-Pacific Journal of Second and Foreign Language Education, 3(15), 1-22. http://doi: 10.1186/s40862-018-0054-2

Zúñiga, E. C. y Gutiérrez, E. R. (2018). Promoting listening fluency in pre-intermediate EFL learners through meaningful oral tasks. Profile Issues in Teachers` Professional Development, 20(2), 161-177. http://doi: 10.15446/profile.v20n2.62938

Fecha de recepción: 5 de mayo de 2020.

Fecha de revisión: 23 de mayo de 2020.

Fecha de aceptación: 20 de noviembre de 2020. 